\title{
REMOCIÓN DE COLORANTES AZO CON ALGINATO: RELACIÓN ENTRE ESTRUCTURA DE COLORANTE Y EFICIENCIA DE REMOCIÓN
}

\author{
Juan Antonio LOZANO-ÁLVAREZ ${ }^{1 *}$, Virginia Francisca MARAÑÓN-RUIZ ${ }^{2}$, Juan JÁUREGUI-RINCÓN ${ }^{1}$, \\ Iliana MEDINA-RAMÍREZ ${ }^{3}$, Rogelio SALINAS-GUTIÉRREZ ${ }^{4}$ y Claudio FRAUSTO-REYES ${ }^{5}$
}

${ }^{1}$ Departamento de Ingeniería Bioquímica, Universidad Autónoma de Aguascalientes, Av. Universidad 940, Ciudad Universitaria, 20131 Aguascalientes, México

${ }^{2}$ Departamento de Ciencias de la Tierra y de la Vida, Centro Universitario de los Lagos, Universidad de Guadalajara. Av. Enrique Díaz de León 1144, Col. Paseos de la Montaña, 47460 Lagos de Moreno, Jalisco, México

${ }^{3}$ Departamento de Química, Universidad Autónoma de Aguascalientes, Av. Universidad 940, Ciudad Universitaria, 20131 Aguascalientes, México

${ }^{4}$ Departamento de Estadística, Universidad Autónoma de Aguascalientes, Av. Universidad 940, Ciudad Universitaria, 20131 Aguascalientes, México

${ }^{5}$ Centro de Investigaciones en Óptica, Unidad Aguascalientes, Prol. Constitución 607, Fracc. Reserva Loma Bonita, 20200 Aguascalientes, México

*Autor de correspondencia: lozanoalvarez@yahoo.com

(Recibido noviembre 2017; aceptado mayo 2018)

Palabras clave: agregación, grupos funcionales, gelificación

\section{RESUMEN}

Los colorantes azo representan entre 60 y $75 \%$ de los colorantes producidos actualmente. Se utilizan ampliamente en la industria cosmética, del papel, optoelectrónica, textil, etc. Desafortunadamente una vez utilizados, debido a su alta resistencia a tratamientos convencionales de aguas residuales, contaminan los cuerpos de agua donde son descargados. En este reporte se utiliza alginato de sodio (ALG) para remover los colorantes azo rojo directo 80 (RD80), rojo congo (Rcongo) y rojo de metilo (Rmetilo). Estudios de espectroscopía UV-visible, FTIR y Raman ayudaron a elucidar los tautómeros involucrados en su remoción. Los porcentajes de remoción fueron: RD80 (99.9\% a $\mathrm{pH}=12$ y fuerza iónica $=0.1 \mathrm{M})$, Rcongo $(99.7 \%$ a pH $=12$ y fuerza iónica $=0.1 \mathrm{M})$ y Rmetilo ( $14.9 \%$ a pH $=7$ y fuerza iónica $=0.1 \mathrm{M})$. El modelo de Zimm-Bragg describe adecuadamente las isotermas experimentales, sugiriendo la importancia de la agregación de los colorantes en su eficiencia de remoción. Adicionalmente, se comparó la eficiencia de remoción de siete colorantes azo y se encontró que moléculas con peso molecular elevado, con alta planaridad y carga positiva mostraron las mayores eficiencias de remoción. Lo anterior permite predecir cualitativamente a partir de las estructuras de colorantes azo, cuál de ellos será removido más eficientemente con el ALG.

Key words: aggregation, functional groups, gelation

\begin{abstract}
The azo dyes represent between $60-75 \%$ of the total of dyes produced nowadays. They are widely used in cosmetic industry, paper, optoelectronic, textile, etc. Unfortunately,
\end{abstract}


due to their high resistance to the conventional treatments applied to wastewaters, they pollute the water bodies where are discharged. In this report, the sodium alginate (ALG) is evaluated for the removal of the azo dyes, Direct red 80 (DR80), Congo red (Rcongo) and Methyl red (Rmetilo). UV-visible, FTIR and Raman spectroscopic studies helped to elucidate the formation of tautomers, species responsible for their removal. The removal percentages were: DR80 $(99.9 \%$ at $\mathrm{pH}=12$ and ionic strength $=0.1 \mathrm{M})$, Rcongo $(99.7 \%$ at $\mathrm{pH}=12$ and ionic strength $=0.1 \mathrm{M})$, and Rmetilo $(14.9 \%$ at $\mathrm{pH}=7$ and ionic strength $=0.1 \mathrm{M}$ ). The Zimm-Bragg model described adequately the experimental isotherms, suggesting the importance of dye's aggregation in their removal efficiency. Additionally, the removal efficiency of seven azo dyes was compared and it was found that the highest removal efficiencies are achieved when dye molecules exhibit high molecular weight, planarity and are positively charged. This allows us to predict in a qualitative way, from the azo dye structures, which of them will be removed more efficiently with ALG.

\section{INTRODUCCIÓN}

Actualmente existe una elevada producción de colorantes sintéticos ya que son utilizados en diversas áreas como la fotográfica, cosmética, del papel, optoelectrónica, teñido de tejidos biológicos, textil, etc. (Freeman y Mock 2012). Del total de colorantes producidos, del 65-70\% son colorantes azo (Gupta y Suhas 2009). Sin embargo, su eliminación representa un problema ambiental debido a que los tratamientos biológicos convencionales no son eficientes en el proceso de degradación de estos compuestos. Lo anterior provoca que al descargarse a diferentes cuerpos de agua, disminuyen la penetración de la luz solar afectando la actividad fotosintética, con la consecuente disminución o agotamiento del oxígeno disuelto, dañando seriamente a las especies acuáticas presentes (Saratale et al. 2011).

Se han utilizado diferentes tratamientos para la remoción de colorantes en efluentes acuosos, entre ellos se encuentran la degradación fotoquímica, ozonización, reactivo de Fenton, fotocatálisis con $\mathrm{TiO}_{2}$, degradación electroquímica, etc. (Srinivasan y Viraraghavan 2010, Oller et al. 2011). Sin embargo, estos métodos tienen algunos inconvenientes como: 1) Una incompleta oxidación de los colorantes generando subproductos como aldehídos, cetonas, ácidos orgánicos e inorgánicos (Robinson et al. 2001). 2) Altos costos para generar ozono y electricidad (Xu et al. 1999). 3) Generación de lodos provenientes de sales de hierro y la inversión en su tratamiento y disposición (Anjaneyulu et al. 2005).

Del mismo modo, la remoción de colorantes mediante el empleo de hongos, bacterias y levaduras requiere condiciones estériles para un desempeño adecuado, lo cual difícilmente se puede mantener en un efluente real (Gupta y Suhas 2009, Solís et al. 2012, Ortiz-Monsalve et al. 2017).

Por otro lado, se han utilizado biopolímeros como la quitina, el quitosano, la xantana y el alginato de sodio nativos y funcionalizados (Bhatnagar y Sillanpää 2009, Lozano-Álvarez et al. 2009, Sadeghi-Kiakhani et al. 2013, Ghorai et al. 2014, Haldorai y Shim 2014, Mittal et al. 2014 y Karadag et al. 2016). Sin embargo, la funcionalización incrementa el costo de producción y disminuye su biodegradabilidad. El alginato de sodio (ALG), es un polisacárido proveniente de algas cafés y bacterias, es utilizado en la industria farmacéutica, de alimentos, de pinturas, etc. como agente espesante y estabilizante. Este compuesto consta de monómeros de $\beta$-D-manuronato y $\alpha$-L-guluronato unidos por enlaces glucosídicos $(1 \rightarrow 4)$. La adición de $\mathrm{Ca}^{+2}$ a una disolución de ALG produce un gel de alginato de calcio, ALG (Ca) (Draget 2009). En este reporte se utiliza dicha propiedad para remover diferentes colorantes azo y se propone una relación entre la estructura del colorante y la eficiencia de remoción por el ALG. Lo anterior proporcionaría una metodología sencilla para definir aproximadamente entre dos o más colorantes azo, cuál de ellos se removerá con mayor eficiencia al utilizar ALG en medio acuoso. Lo cual es de interés ambiental, dada la variabilidad de estructuras de estos colorantes presentes en descargas de agua contaminada.

\section{MATERIALES Y MÉTODOS}

El rojo directo 80 (RD80), rojo congo (Rcongo), rojo de metilo (Rmetilo) y el ALG fueron adquiridos de Sigma-Aldrich. El NaOH, $\mathrm{HCl}, \mathrm{NaCl}$ y $\mathrm{CaCl}_{2}$ son de marca J.T. Baker. 
Estudios de espectroscopía ultra violeta visible Se obtuvieron bajo las siguientes condiciones:

\section{Efecto del pH}

Se prepararon disoluciones acuosas de cada colorante $\left(1 \times 10^{-5} \mathrm{M}\right)$ a diferentes valores de $\mathrm{pH}$ (4-12) y se obtuvo su espectro de absorción ultra violeta visible (UV-visible).

Efecto de la concentración del colorante en presencia y ausencia del ALG

Se prepararon disoluciones de cada colorante a diferentes concentraciones $(5-100 \mathrm{mg} / \mathrm{L})$ en las condiciones de $\mathrm{pH}$ y fuerza iónica (F.I.) con mayor eficiencia de remoción para cada colorante. Lo anterior se realizó en ausencia y en presencia del ALG al $1 \%$ peso volumen $(\mathrm{p} / \mathrm{v})$ para observar el efecto de este polímero en la agregación del colorante. En el caso de este experimento y el siguiente, se modificó el paso de luz de la celda para ajustar la absorbancia al límite máximo de detección del espectrofotómetro, por ende, la absorbancia no es lineal con la concentración de colorante.

\section{Efecto del agente gelificante}

Se realizó el procedimiento descrito en el párrafo anterior para obtener el producto ALG-colorante, pero adicionalmente se añadió $\mathrm{CaCl}_{2}$ al $5 \%$ peso volumen $(\mathrm{p} / \mathrm{v})$ de manera posterior, para obtener el gel de ALG-colorante ( $\mathrm{Ca})$.

Estudios de espectroscopía de absorción en el infrarrojo medio con transformada de Fourier (FTIR) y de dispersión Raman

Se preparó el producto ALG-colorante $(\mathrm{Ca})$ en polvo, de acuerdo con lo reportado previamente por Jáuregui-Rincón et al. (2011). Los estudios de espectroscopía de absorción en el infrarrojo medio con transformada de Fourier (FTIR) se realizaron en un equipo Jasco modelo 4600 series en modo de reflectancia total atenuada $\left(4000-600 \mathrm{~cm}^{-1}\right)$ con una ventana de diamante. Para la obtención del espectro Raman (2000-400 $\left.\mathrm{cm}^{-1}\right)$ se utilizó un equipo MicroRaman system modelo Renishaw 1000.

\section{Optimización de las condiciones de remoción de colorantes}

Se prepararon disoluciones de colorante que incluyeran al ALG hidratado (concentración final de $0.5 \% \mathrm{p} / \mathrm{v}$ ) y se les ajustó los valores de $\mathrm{pH}$ y F.I. para cada colorante, de tal manera que los valores de concentración y volumen finales fueron $100 \mathrm{mg} / \mathrm{L}$ y $100 \mathrm{~mL}$ respectivamente (Jáuregui-Rincón et al.
2011). Debido a la baja solubilidad mostrada por el Rmetilo a valores de $\mathrm{pH}$ inferiores a 7, los valores de $\mathrm{pH}$ utilizados fueron 7, 9.5 y 12 con una F.I. de $0.1 \mathrm{M}, 0.5 \mathrm{M}$ y $0.9 \mathrm{M}$. Tras la gelificación con 30 $\mathrm{mL}$ de $\mathrm{CaCl}_{2}$ al $5 \% \mathrm{p} / \mathrm{v}$, se aforó con agua destilada a $200 \mathrm{~mL}$. Después de reposar $24 \mathrm{~h}$ en la oscuridad, el sobrenadante se centrifugó a $9000 \mathrm{~g}$ (20 min). La concentración del colorante se determinó por espectroscopía UV-visible en un espectrofotómetro Thermo Scientific modelo Evolution 201 (todos los experimentos se realizaron por triplicado).

El porcentaje de remoción se obtuvo con la fórmula:

$\%$ Remoción $=(C i-C e) \times 100 / C i$

Donde:

$\mathrm{Ci}$ y $\mathrm{Ce}$ son las concentraciones inicial y final del colorante $(\mathrm{mol} / \mathrm{L})$, respectivamente.

\section{Obtención de las isotermas de adsorción}

Se prepararon disoluciones de colorante con diferentes concentraciones $(0-2000 \mathrm{mg} / \mathrm{L})$ con los valores de $\mathrm{pH}$ y F.I. ajustados de acuerdo con lo obtenido en el apartado anterior y se adicionó el ALG $(0.5 \mathrm{~g})$. Posteriormente se gelificó con $\mathrm{CaCl}_{2}$ al $5 \% \mathrm{p} / \mathrm{v}$ y se cuantificó la concentración de colorante como se describió previamente (ver sección anterior).

\section{Aplicación del modelo de Zimm-Bragg a las iso- termas experimentales}

La aplicación de este modelo a los datos experimentales se realizó de la misma manera como previamente lo hicieron Lozano-Álvarez et al. (2009) y Jáuregui-Rincón et al. (2011). Se determinó el parámetro qe (cantidad de colorante en moles removida por mol de ALG) mediante la siguiente fórmula:

$q e=(C i-C e) \times V / m$

\section{Dónde:}

Ci y $\mathrm{Ce}$ se definieron anteriormente, $\mathrm{V}=$ volumen de colorante (L) y $\mathrm{m}=$ masa de ALG (mol).

Los parámetros teóricos del modelo de ZimmBragg (1959) se obtuvieron utilizando el paquete estadístico R (Core 2013), introduciendo la función de este modelo y los valores iniciales de los parámetros $K_{\mathrm{u}}$ y $U$ para iniciar el proceso de optimización. Dicho proceso utilizó la siguiente función como parámetro de optimización:

$L\left(K_{u}, U\right)=\sum_{i=1}^{n}\left(\frac{\beta_{i}-\beta_{t}}{\beta_{i}}\right)^{2}$ 
Dónde: $\beta i$ son los valores experimentales obtenidos en la isoterma experimental y $\beta t$ son los valores teóricos obtenidos por el proceso de optimización.

El paquete finalizó el proceso de optimización cuando el valor de L fue mínimo. Finalmente se obtuvieron los valores de $K_{\mathrm{u}}$ y $U$ para cada sistema, el coeficiente de determinación $\left(\mathrm{R}^{2}\right)$ y el error relativo promedio.

\section{RESULTADOS Y DISCUSIÓN}

\section{Estudios de espectroscopía ultravioleta visible}

La presencia de grupos funcionales como el hidroxilo $(-\mathrm{OH})$, el amino $\left(-\mathrm{NH}_{2}\right)$ y el carboxilo $(-\mathrm{COOH})$ en colorantes azo produce diferentes efectos al cambiar el pH. En el caso del RD80 se observa un equilibrio tautomérico azo-hidrazona, que favorece a la forma hidrazona cuando el -OH se encuentra en posición orto con respecto al grupo azo (Ozen et al. 2007, Abbott et al. 2011) (Fig. 1). Lo anterior se evidencia por la banda localizada en $530 \mathrm{~nm}(\mathrm{pH}$ $=4)$ hasta $540(\mathrm{pH}=11)$ en el espectro ultravioleta visible del RD80. Más aún, Oakes y Gratton (1998) reportaron la existencia de un "anión común" a valores de $\mathrm{pH}$ entre 12 y 14, que para el RD80 está ubicado en $545 \mathrm{~nm}$.

La formación de agregados tipo $\mathrm{H}$ de colorantes azo debido al apilamiento de sus anillos aromáticos se ha manifestado como un desplazamiento hipsocrómico en el $\lambda_{\max }$ del espectro UV-visible del colorante (Karukstis et al. 2002, Song et al. 2017). En este sentido el espectro UV-visible del RD80 a $\mathrm{pH}=12$ fue similar a diferentes concentraciones (5-
$200 \mathrm{mg} / \mathrm{L}$, datos no mostrados), sugiriendo que no existió una agregación inducida por el incremento en la concentración de este colorante. Adicionalmente el espectro UV-visible del RD80 a pH = 12 fue similar en presencia y ausencia del ALG en disolución acuosa $\left(\lambda_{\max }=545 \mathrm{~nm}\right)($ Fig. 2 a-b), indicando que el RD80 presenta el mismo grado de agregación en ambos casos y que la fuerza de interacción entre ellos es tal que no afecta la estructura electrónica del RD80. Esto contrasta con lo observado por Pal y Mandal (1990), donde se encontró que la adición de alginato de potasio "fragmentó" los agregados de pinacianol y se observó un desplazamiento batocrómico de su $\lambda_{\max }$.

Por otra parte, la adición de $\mathrm{CaCl}_{2}$ al ALGRD80, formó el gel de ALG-RD80 (Ca) y ocurrió un

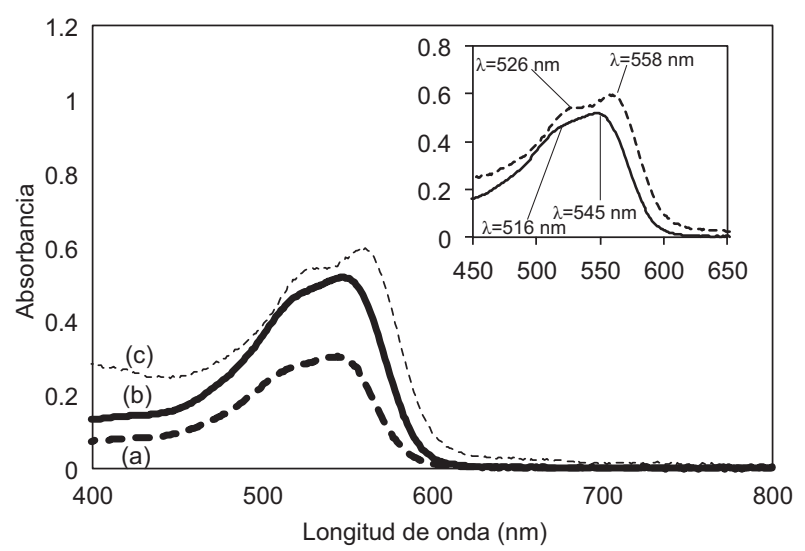

Fig. 2. Espectro de absorción electrónica del DR80 (a) y del producto ALG-DR80 (b), ambos en disolución acuosa y del gel de ALG-DR80 (Ca) (c). En el recuadro superior derecho se muestran a detalle los espectros (b) y (c)

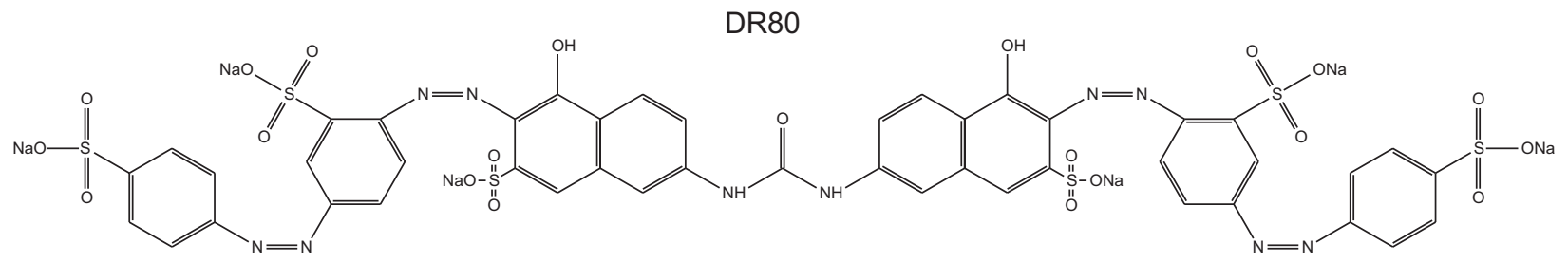<smiles>[R20]CC1CCC(C2CCC(NNC3CC(S(O)(O)O)C4CCCCC4C3N)CC2)CC1NNC1CC(S(O)(O)O)C2CCCCC2C1N</smiles><smiles>CN(O)C1CCC(NNC2CCCCC2C(O)ON)CC1</smiles>

Fig.1. Estructura de los colorantes utilizados en este estudio 
desdoblamiento en los máximos ubicados en $\lambda_{\max }=$ $516 \mathrm{~nm}$ y $\lambda_{\max }=545 \mathrm{~nm}$ hasta $\lambda_{\max }=526 \mathrm{~nm}$ y $\lambda_{\max }$ $=558 \mathrm{~nm}$ respectivamente (ver recuadro superior derecho, Fig. 2). Esto sugiere que la adición de $\mathrm{Ca}^{+2}$ a la disolución de ALG-RD80 atrae las moléculas del RD80 (que poseen carga negativa a $\mathrm{pH}=12$ ), favoreciendo la desagregación del RD80 adsorbido al ALG. Sin embargo, al formarse el gel de ALGRD80 (Ca) tales moléculas son "atrapadas" dentro de él y en consecuencia la concentración del RD80 en la disolución acuosa disminuye (Lozano-Alvarez et al. 2015).

Se ha reportado que moléculas de colorante azo más grandes, con anillos aromáticos extendidos y grupos funcionales que favorecen su planaridad, forman agregados más estables como consecuencia del apilamiento $\pi$ - $\pi$ (Oakes y Dixon 2004). La figura 1 muestra que el RD80 cumple con estas características, peso molecular $(\mathrm{PM})=1373.07 \mathrm{~g} / \mathrm{mol}$, contiene cuatro grupos fenilo y dos naftilo, resultando en la formación de agregados de RD80 cuya existencia sólo se evidencia al producirse el ALG-RD80 (Ca).

Los espectros UV-visible del Rcongo en disolución acuosa $\left(1 \times 10^{-5} \mathrm{M}\right)$ a valores de $\mathrm{pH}=4$ presentan un color azul morado debido a la protonación de este colorante que posee un $\mathrm{pKa}=4.1$ (Sabnis 2007). Lo anterior da como resultado las formas quinoide y azonio reportadas por Elhaddaoui et al. (1992), Bonancea et al. (2006) y Barkauskas et al. (2011), que se manifiestan como una banda con $\lambda_{\max }=515 \mathrm{~nm}$ y un hombro ancho que se extiende hasta los $800 \mathrm{~nm}$. A pH $=6, \lambda_{\max }$ se desplaza hasta $498 \mathrm{~nm}$ (disolución naranja), debido a la existencia de la forma neutra que incluye al grupo azo en su estructura (Movahedi et al. 2009). Incrementos posteriores en el $\mathrm{pH}$ resultan en una $\lambda_{\max }=498$ $\mathrm{nm}$, observándose cambios ligeros en la intensidad debido a la desprotonación del Rcongo. Sólo hasta llegar a un $\mathrm{pH}=12$ se observa un pequeño cambio en $\lambda_{\max },(494 \mathrm{~nm})$. El Rcongo tiende a apilarse en concentraciones de $\mathrm{NaCl}$ superiores al $0.3 \%$ (0.05 M) (Han et al. 2008). Experimentalmente se utilizó una F.I. mayor para obtener los espectros UV-visible (F.I. $=0.1 \mathrm{M})$, lo cual sugiere que existe una agregación de las moléculas del Rcongo por interacción $\pi-\pi$ de sus grupos naftilo, concordando con la formación de estructuras supramoleculares reportadas por Skowronek et al. (1998). Lo anterior se corroboró al observar un desplazamiento hipsocrómico de $\lambda_{\max }=488 \mathrm{~nm}(5 \mathrm{mg} / \mathrm{L})$ hasta $\lambda_{\max }=$ $485 \mathrm{~nm}(100 \mathrm{mg} / \mathrm{L})$ a $\mathrm{pH}=12$. Los espectros UVvisible del Rcongo en presencia y en ausencia del ALG resultaron muy semejantes. Lo anterior sugiere que este polisacárido no promueve una agregación adicional del Rcongo a $\mathrm{pH}=12$, por la repulsión existente entre ambas moléculas, evitando que las moléculas del colorante se agreguen en la superficie molecular del ALG.

La adición del ion $\mathrm{Ca}^{+2}$ a la disolución de ALGRcongo ocasionó un desplazamiento batocrómico desde $\lambda_{\max }=485 \mathrm{~nm}$ a $\lambda_{\max }=488 \mathrm{~nm}$, sugiriendo una desagregación del Rcongo similar a la ocurrida con el RD80, pero con menor intensidad debido a la menor carga negativa del Rcongo comparada con aquella del RD80 (Fig. 1). Es decir, el efecto del $\mathrm{Ca}^{+2}$ promueve una ligera desagregación, pero la formación del gel "atrapa" a las moléculas y agregados del Rcongo aún adsorbidos a la superficie molecular del ALG. Lo anterior favorece la retención de este colorante en el producto ALG-Rcongo $(\mathrm{Ca})$, resultando una eficiencia de remoción del $99.7 \%$ (similar a la encontrada con el $\mathrm{RD} 80=99.9 \%$, ambos a $\mathrm{pH}=12$ y F.I. $=0.1 \mathrm{M}$ ).

Se obtuvieron espectros UV-visible del Rmetilo de experimentos similares a los realizados con los otros colorantes. Park et al. (2004) han reportado que en el intervalo de $\mathrm{pH}=4$ a 6 existen los mayores cambios en la distribución electrónica del Rmetilo y que se deben a la existencia de las especies aniónica y protonada de este compuesto, dado que su pKa es de 5.02 (García-Heras et al. 2005). Experimentalmente se encontró que valores menores y mayores a este $\mathrm{pH}$ produjeron espectros de absorción electrónica muy diferentes, a $\mathrm{pH}=4, \lambda_{\max }=525 \mathrm{~nm}$ (disolución color rojo) y a $\mathrm{pH}=6, \lambda_{\max }=431 \mathrm{~nm}$ (disolución color naranja). Incrementos posteriores en el $\mathrm{pH}$ disminuyeron la intensidad del color de la disolución sin un cambio importante en $\lambda_{\max }$ por el predominio de la forma aniónica del colorante.

Cuando se puso en contacto al ALG con el Rmetilo (5-100 mg/L), se observó que los espectros UVvisible fueron semejantes a los obtenidos solamente con el colorante $\left(\lambda_{\max }=431 \mathrm{~nm}\right)$, sugiriendo que el Rmetilo tiene poca tendencia a agregarse y que el ALG no promueve su agregación. Esto indica que las interacciones entre ambas moléculas son débiles (puentes de hidrógeno e interacciones hidrofóbicas) (Blackburn 2004) y que aunque existe una interacción electrostática entre el grupo amino terciario del Rmetilo con los grupos carboxilo del ALG, no es detectable por espectroscopía UV-visible. Tras la adición del ion $\mathrm{Ca}^{+2}$, no hubo cambios en $\lambda_{\max }$ (431 $\mathrm{nm})$, indicando que la formación del ALG-Rmetilo (Ca) tiene poco efecto en el espectro de absorción electrónica, por la agregación mínima del Rmetilo en dicho compuesto. 


\section{Estudios de espectroscopía FTIR y dispersión Raman}

Para entender de manera más profunda la interacción del ALG con los colorantes, se obtuvieron espectros de absorción FTIR y Raman. El cuadro I incluye las señales espectroscópicas más relevantes del RD80, ALG (Ca) y el ALG-RD80 (Ca). Este cuadro muestra que los grupos hidroxilo presentes en el RD80 forman puentes de hidrógeno (banda ancha centrada en $\left.3390 \mathrm{~cm}^{-1}\right)$, lo cual dificulta encontrar las vibraciones $v(\mathrm{~N}-\mathrm{H})$ del grupo amida y $v(\mathrm{C}-\mathrm{H})$ de los anillos aromáticos de este colorante. Sin embargo, las vibraciones $v(\mathrm{C}=\mathrm{O})$ del grupo amido (1647 $\left.\mathrm{cm}^{-1}\right)$ y $v(\mathrm{C}=\mathrm{N})\left(1565 \mathrm{~cm}^{-1}\right)$ indican que el tautómero hidrazona está presente en este colorante (Schrader 1995). Por otra parte, la vibración $v(\mathrm{C}-\mathrm{O})$ típica de un alcohol aromático presente en $1000 \mathrm{~cm}^{-1}$ sugiere que la forma azo del RD80 también está presente en estado sólido (Silverstein et al. 2015).
El cuadro I muestra que los espectros del ALG (Ca) y ALG-RD80 (Ca) son muy similares y que las señales características del RD80 descritas anteriormente no se pueden identificar fácilmente en el ALG-RD80 (Ca). Así, los puentes de hidrógeno del RD80 se traslaparon con aquellos generados por los grupos hidroxilo presentes en el ALG (Ca), observándose una banda ancha localizada en $3285 \mathrm{~cm}^{-1}$ en ambos compuestos. Las vibraciones $v_{\text {asym }}(\mathrm{COO})$ y $v_{\mathrm{sym}}(\mathrm{COO})$ encontradas en $1591 \mathrm{~cm}^{-1}$ y $1407 \mathrm{~cm}^{-1}$ respectivamente, sugieren que el "ambiente químico" experimentado por el grupo carboxilo es similar en ambos compuestos. Sin embargo, la vibración $v_{\text {asym }}(\mathrm{COO})$ dificultó observar las vibraciones $v(\mathrm{C}=\mathrm{O})$ y $v(\mathrm{C}=\mathrm{N})$ de la forma hidrazona del RD80. Del mismo modo, la vibración $v(\mathrm{C}-\mathrm{O})$ ubicada en $1028 \mathrm{~cm}^{-1}$ típica de polisacáridos se traslapa con la misma vibración del colorante.

CUADRO I. DATOS DE ESPECTROSCOPÍA DE ABSORCIÓN EN EL INFRARROJO MEDIO CON TRANSFORMADA DE FOURIER (FTIR) Y RAMAN DE LOS COMPUESTOS ALGINATO DE CALCIO: ALG (Ca), DR80 y ALG-DR80 (Ca)

\begin{tabular}{|c|c|c|c|c|}
\hline $\begin{array}{l}\text { Señales } \\
\text { FTIR }\end{array}$ & ALG (Ca) & DR80 & ALG-DR80 (Ca) & Observaciones \\
\hline$v(\mathrm{OH})$ & $3285 \mathrm{~cm}^{-1}$ & $3390 \mathrm{~cm}^{-1}$ & $3285 \mathrm{~cm}^{-1}$ & $\begin{array}{l}\text { Los puentes de hidrógeno existentes en el DR80 se } \\
\text { traslapan con los encontrados en el ALG }(\mathrm{Ca}) \text { y se } \\
\text { incrementa la anchura de la banda. }\end{array}$ \\
\hline$v(\mathrm{C}-\mathrm{H})$ & & $3100 \mathrm{~cm}^{-1}$ & No se detecta & $\begin{array}{l}\text { Los puentes de hidrógeno existentes en el ALG }(\mathrm{Ca}) \\
\text { evitan la detección de esta señal en el ALG-DR80 (Ca). }\end{array}$ \\
\hline$v_{\text {asym }}(\mathrm{COO})$ & $1591 \mathrm{~cm}^{-1}$ & & $1591 \mathrm{~cm}^{-1}$ & $\begin{array}{l}\text { El ambiente químico del grupo carboxilo es muy } \\
\text { semejante en el ALG (Ca) y en el ALG-DR80 (Ca). }\end{array}$ \\
\hline$v_{\text {sym }}(\mathrm{COO})$ & $1407 \mathrm{~cm}^{-1}$ & & $1407 \mathrm{~cm}^{-1}$ & $\begin{array}{l}\text { El grupo carboxilo experimenta un "ambiente químico" } \\
\text { similar en el ALG (Ca) y en el ALG-DR80 (Ca). }\end{array}$ \\
\hline$v(\mathrm{C}-\mathrm{O})$ & $1028 \mathrm{~cm}^{-1}$ & $1000 \mathrm{~cm}^{-1}$ & $1028 \mathrm{~cm}^{-1}$ & La señal del colorante está inmersa en la del ALG $(\mathrm{Ca})$. \\
\hline$v(\mathrm{C}=\mathrm{O})$ de amida & & $1647 \mathrm{~cm}^{-1}$ & No se detecta & $\begin{array}{l}\text { La vibración } v(\mathrm{C}=\mathrm{O}) \text { de la amida del } \mathrm{DR} 80 \text { se traslapa } \\
\text { con la señal } v_{\text {asym }}(\mathrm{COO}) \text { del ALG }(\mathrm{Ca})\end{array}$ \\
\hline$v(\mathrm{C}=\mathrm{N})$ de hidrazona & & $1565 \mathrm{~cm}^{-1}$ & No se detecta & $\begin{array}{l}\text { La vibración } v(\mathrm{C}=\mathrm{N}) \text { de la amida del } \mathrm{DR} 80 \text { se traslapa } \\
\text { con la señal } v_{\text {asym }}(\mathrm{COO}) \text { del ALG }(\mathrm{Ca}) \text { en el aducto } \\
\text { ALG-DR80 }(\mathrm{Ca}) \text {. }\end{array}$ \\
\hline
\end{tabular}

Señales Raman

\begin{tabular}{|c|c|c|c|}
\hline $\begin{array}{l}v(\mathrm{C}=\mathrm{C}) \text { de grupos } \\
\text { fenilo }\end{array}$ & $1594 \mathrm{~cm}^{-1}$ & $1594 \mathrm{~cm}^{-1}$ & $\begin{array}{l}\text { Esta señal confirma la presencia del DR80 en el ALG- } \\
\text { DR80 (Ca). }\end{array}$ \\
\hline $\begin{array}{l}v(\mathrm{C}=\mathrm{N}) \text { de tautómero } \\
\text { hidrazona }\end{array}$ & $1565 \mathrm{~cm}^{-1}$ & $1563 \mathrm{~cm}^{-1}$ & $\begin{array}{l}\text { El tautómero hidrazona se encuentra en ambos } \\
\text { compuestos (DR80 y ALG-DR80 (Ca)). }\end{array}$ \\
\hline $\begin{array}{l}v(\mathrm{~N}=\mathrm{N}) \text { de tautómero } \\
\text { azo }\end{array}$ & $1448 \mathrm{~cm}^{-1}$ & $1449 \mathrm{~cm}^{-1}$ & $\begin{array}{l}\text { El tautómero azo se encuentra en ambos compuestos } \\
(\mathrm{DR} 80 \text { y ALG-DR80 (Ca)). }\end{array}$ \\
\hline$v\left(-\mathrm{SO}_{3}\right)$ & \multicolumn{2}{|c|}{1174 y $1154 \mathrm{~cm}^{-1} 1178$ y $1155 \mathrm{~cm}^{-1}$} & $\begin{array}{l}\text { Esta señal confirma la presencia del DR80 en el ALG- } \\
\text { DR80 }(\mathrm{Ca}) .\end{array}$ \\
\hline
\end{tabular}


Las señales del espectro Raman del RD80 y el ALG-RD80 (Ca) se incluyen en el cuadro I, cabe mencionar que la fluorescencia mostrada por el ALG (Ca) fue demasiado alta y dificultó la obtención de su espectro Raman. La vibración $v(\mathrm{C}=\mathrm{C})$ de grupos fenilo $\left(1594 \mathrm{~cm}^{-1}\right)$ está presente en ambos compuestos, sugiriendo que el ambiente electrónico de los anillos bencénicos es similar en los dos compuestos. Las señales localizadas en $1565 \mathrm{~cm}^{-1}$ y $1448 \mathrm{~cm}^{-1}$ correspondientes a las vibraciones $v(\mathrm{C}=\mathrm{N})$ y $v(\mathrm{~N}=\mathrm{N})$ de las formas hidrazona y azo respectivamente, corroboran su presencia en el colorante en polvo y en el ALG-RD80 (Ca) (Armstrong et al. 1995, Biswas y Umapathy 2000). Adicionalmente, las señales características de los grupos sulfonato del colorante $\left(1174 \mathrm{~cm}^{-1}\right.$ y $\left.1154 \mathrm{~cm}^{-1}\right)$, también se encontraron en el ALG-RD80 (Ca).

El cuadro II resume las señales más importantes de los espectros FTIR y Raman del ALG (Ca), Rcongo y ALG-Rcongo (Ca). Dichas señales muestran que los picos característicos del Rcongo se traslapan con aquellos del ALG (Ca), de tal manera que el espectro del ALG-Rcongo (Ca) es similar al espectro del ALG $(\mathrm{Ca})$. Por ejemplo, la vibración $v(\mathrm{OH})$ $\left(3290 \mathrm{~cm}^{-1}\right)$ es amplia y dificulta la observación de las señales $v(\mathrm{C}-\mathrm{H})$ de los anillos aromáticos del Rcongo $\left(3075 \mathrm{~cm}^{-1}\right)$ y las señales del grupo amino $v_{\text {asym }}(\mathrm{N}-\mathrm{H})$ y $v_{\text {sym }}(\mathrm{N}-\mathrm{H})\left(3460 \mathrm{~cm}^{-1}\right.$ y $3350 \mathrm{~cm}^{-1}$, respectivamente). Del mismo modo, las señales $v(\mathrm{C}-\mathrm{N})$ y $\delta(\mathrm{N}-\mathrm{H})\left(1346 \mathrm{~cm}^{-1}\right.$ y $1583 \mathrm{~cm}^{-1}$, respectivamente) del grupo amino unido al grupo naftilo, son difíciles de localizar en el espectro del ALG-Rcongo (Ca) debido a que las vibraciones $v_{\text {asym }}(\mathrm{COO})$ y $v_{\text {sym }}(\mathrm{COO})$ $\left(1595 \mathrm{~cm}^{-1}\right.$ y $1414 \mathrm{~cm}^{-1}$, respectivamente) dificultan su identificación.

Por otro lado, el espectro Raman mostró claramente la presencia del Rcongo en el ALG-Rcongo (Ca). Por ejemplo, la vibración $v(\mathrm{C}=\mathrm{C})$ típica de los

CUADRO II. DATOS DE ESPECTROSCOPÍA DE ABSORCIÓN EN EL INFRARROJO MEDIO CON TRANSFORMADA DE FOURIER (FTIR) Y RAMAN DE LOS COMPUESTOS ALGINATO DE CALCIO: ALG (Ca), Rcongo y ALG-Rcongo (Ca)

\begin{tabular}{|c|c|c|c|c|}
\hline $\begin{array}{l}\text { Señales } \\
\text { FTIR }\end{array}$ & ALG (Ca) & Rcongo & ALG-Rcongo (Ca) & Observaciones \\
\hline$v(\mathrm{OH})$ & $3290 \mathrm{~cm}^{-1}$ & & $3290 \mathrm{~cm}^{-1}$ & $\begin{array}{l}\text { Los puentes de hidrógeno debido al grupo hidroxilo se encuentran } \\
\text { presentes en el ALG-Rcongo }(\mathrm{Ca}) \text {. }\end{array}$ \\
\hline$v_{\text {asym }}(\mathrm{COO})$ & $1594 \mathrm{~cm}^{-1}$ & & $1595 \mathrm{~cm}^{-1}$ & $\begin{array}{l}\text { El "ambiente químico" del grupo carboxilo del ALG es el mismo } \\
\text { en el ALG }(\mathrm{Ca}) \text { y en el ALG-Rcongo }(\mathrm{Ca}) \text {. }\end{array}$ \\
\hline$v_{\text {sym }}(\mathrm{COO})$ & $1414 \mathrm{~cm}^{-1}$ & & $1414 \mathrm{~cm}^{-1}$ & $\begin{array}{l}\text { El grupo carboxilo experimenta un "ambiente químico" similar } \\
\text { en el ALG }(\mathrm{Ca}) \text { y en el ALG-DR80 (Ca). }\end{array}$ \\
\hline$v(\mathrm{C}-\mathrm{N})$ & & $1346 \mathrm{~cm}^{-1}$ & No se detecta & $\begin{array}{l}\text { La vibración } v(\mathrm{C}-\mathrm{N}) \text { del grupo amino del Rcongo se traslapa con } \\
\text { la señal } v_{\text {sym }}(\mathrm{COO}) \text { del ALG }(\mathrm{Ca}) \text {. }\end{array}$ \\
\hline$\delta(\mathrm{N}-\mathrm{H})$ & & $1583 \mathrm{~cm}^{-1}$ & No se detecta & Esta señal está inmersa en la vibración $v_{\text {asym }}(\mathrm{COO})$ del ALG $(\mathrm{Ca})$. \\
\hline $\begin{array}{l}v(\mathrm{C}-\mathrm{H}) \text { de anillo } \\
\text { aromático }\end{array}$ & & $3075 \mathrm{~cm}^{-1}$ & No es detectable & $\begin{array}{l}\text { Los puentes de hidrógeno del grupo hidroxilo presentes en el } \\
\text { ALG-Rcongo }(\mathrm{Ca}) \text { evitan encontrar esta vibración. }\end{array}$ \\
\hline$v_{\text {asym }}(\mathrm{N}-\mathrm{H})$ & & $3460 \mathrm{~cm}^{-1}$ & No se detecta & $\begin{array}{l}\text { La banda amplia debida a los puentes de hidrógeno del grupo } \\
\text { hidroxilo presentes en el ALG-Rcongo }(\mathrm{Ca}) \text { evita detectar estas } \\
\text { vibraciones. }\end{array}$ \\
\hline$v_{\text {sym }}(\mathrm{N}-\mathrm{H})$ & & $3350 \mathrm{~cm}^{-1}$ & No se detecta & Igual que para el caso de la vibración $v_{\text {asym }}(\mathrm{N}-\mathrm{H})$. \\
\hline \multicolumn{5}{|l|}{ Señales Raman } \\
\hline $\begin{array}{l}v(\mathrm{C}=\mathrm{C}) \mathrm{de} \\
\text { grupos fenilo }\end{array}$ & & $1590 \mathrm{~cm}^{-1}$ & $1592 \mathrm{~cm}^{-1}$ & $\begin{array}{l}\text { Esta señal confirma la presencia del Rcongo en el ALG-Rcongo } \\
\text { (Ca). }\end{array}$ \\
\hline$v(\mathrm{~N}=\mathrm{N})$ & & $1450 \mathrm{~cm}^{-1}$ & $1450 \mathrm{~cm}^{-1}$ & $\begin{array}{l}\text { Esta señal confirma la presencia del grupo azo en el Rcongo y } \\
\text { en el ALG-Rcongo }(\mathrm{Ca}) \text {. }\end{array}$ \\
\hline$v\left(-\mathrm{SO}_{3}\right)$ & & $1154 \mathrm{~cm}^{-1}$ & $1156 \mathrm{~cm}^{-1}$ & $\begin{array}{l}\text { Esta señal confirma la presencia del Rcongo en el ALG-Rcongo } \\
\text { (Ca). }\end{array}$ \\
\hline
\end{tabular}


grupos fenilo del Rcongo $\left(1590 \mathrm{~cm}^{-1}\right)$, aparece en $1592 \mathrm{~cm}^{-1}$ en el ALG-Rcongo (Ca). Igualmente, la vibración $v(\mathrm{~N}=\mathrm{N})$ se encontró en el espectro de ambos compuestos en $1450 \mathrm{~cm}^{-1}$ y los grupos sulfonato del colorante $\left(1154 \mathrm{~cm}^{-1}\right)$, también se encontraron en el ALG-Rcongo (Ca) $\left(1156 \mathrm{~cm}^{-1}\right)$.

El FTIR del Rmetilo indica que se encuentra como sal sódica, debido a las vibraciones $v_{\text {asym }}(\mathrm{COO}) \mathrm{y}$ $v_{\text {sym }}(\mathrm{COO})$ ubicadas en $1595 \mathrm{~cm}^{-1}$ y $1441 \mathrm{~cm}^{-1}$, respectivamente. La señal $v_{\text {arom }}(\mathrm{C}-\mathrm{H}$ presente en $\left.3286 \mathrm{~cm}^{-1}\right)$ sugiere la presencia de anillos aromáticos y la vibración $v(\mathrm{C}-\mathrm{N})$ de la amina terciaria aparece en $1362 \mathrm{~cm}^{-1}$. De modo similar a los otros colorantes, algunos grupos funcionales del ALG $(\mathrm{Ca})$ dificultan la detección de las señales correspondientes al Rmetilo. Por ejemplo, la señal originada por la vibración $v(\mathrm{C}-\mathrm{H})$ no se detectó en el espectro del ALG-Rmetilo (Ca) debido a que se encuentra inmersa en la banda típica de la vibración $v(\mathrm{OH})$ del ALG $(\mathrm{Ca})$. Del mismo modo, los picos característicos del grupo carboxilato del Rmetilo también se traslaparon con las señales del mismo grupo funcional del ALG (Ca). Finalmente, la concentración del colorante en el ALG-Rmetilo (Ca) fue demasiado baja para lograr diferenciar los picos de sus grupos funcionales del ruido presente en su espectro Raman.

\section{Optimización de las condiciones de remoción de colorantes}

Los estudios cinéticos realizados previamente con disoluciones de $100 \mathrm{mg} / \mathrm{L}$ de colorante (datos no mostrados), indicaron que el equilibrio se alcanza a las dos horas. Además, las constantes de velocidad $\left(\mathrm{K}_{2}\right)$ obtenidas al aplicar el modelo de pseudo segundo orden (Ho y McKay 1999), fueron las siguientes: $0.1117 \mathrm{~g} / \mathrm{min} \mathrm{mg}$ (RD80), $0.039200 \mathrm{~g} / \mathrm{min} \mathrm{mg}$ (Rcongo) y $0.06077 \mathrm{~g} / \mathrm{min} \mathrm{mg}$ (Rmetilo). Esta información sugiere que la quimioadsorción está involucrada en la remoción de estos colorantes y permitió utilizar un intervalo de tiempo adecuado para los estudios de optimización. La figura 3a muestra la influencia del pH y F.I. en la eficiencia de remoción del RD80. Se observa que al incrementarse el $\mathrm{pH}$, la remoción también lo hace, debido a un incremento en la solubilidad del colorante por la desprotonación de sus grupos ionizables y en consecuencia su interacción con el ALG. Contrariamente, al disminuir la F.I. el ALG remueve más RD80, sugiriendo que aunque existe una repulsión considerable entre las moléculas del RD80 y el ALG, hay interacciones que favorecen la unión entre ellas. Los grupos funcionales existentes en el RD80 (hidroxilo, amido, azo y sulfonato) forman puentes de hidrógeno con los grupos oxhidrilo, carboxilo y con el oxígeno de los enlaces hemiacetálico y glucosídicos del ALG. Adicionalmente, Clark (2011) reportó que la existencia de interacciones hidrofóbicas entre los electrones $\pi$ de los anillos aromáticos del colorante y los grupos $\mathrm{OH}$ de la celulosa promueven una mayor fijación del colorante a este polisacárido. Debido a la semejanza del ALG con la celulosa, es evidente que esta interacción contribuye considerablemente a la unión del RD80 con el ALG. El incremento de electrolitos disminuye la eficiencia de remoción debido a que los iones interaccionan con los grupos funcionales con carga opuesta presentes en el RD80 (sulfonato e hidroxilo desprotonados) y con el carboxilo e hidroxilo desprotonados del ALG, disminuyendo la interacción entre ambas moléculas. Lo anterior arrojó una remoción del $99.9 \%$ a $\mathrm{pH}=$ 12 y F.I. $=0.1 \mathrm{M}$.

Cuando se remueve el Rcongo con ALG, la figura 3b sugiere que existen dos zonas en las cuales la capacidad de remoción es máxima: $\mathrm{pH}$ bajo $(\mathrm{pH}=$ 4) y pH alto $(\mathrm{pH}=12)$. Sin embargo, es importante mencionar que a $\mathrm{pH}=4$ el Rcongo se precipita, por la proximidad con el $\mathrm{pKa}=4.1$ de este colorante (Sabnis 2007). En este sentido se podría decir que el ALG no remueve al colorante disuelto, sino al precipitado, lo cual sobreestimaría la eficiencia de remoción y dificultaría el modelado de los datos experimentales. Además, se observó que a valores de F.I. $=0.9 \mathrm{M}$, una parte considerable del Rcongo se insolubilizó (a pH $=12 \mathrm{y} \mathrm{pH}=4$ se observó una mayor precipitación), lo cual generó el mínimo observado en la figura $\mathbf{3 b}$ a pH de 8.0. Así, el Rcongo sólo permaneció soluble a $\mathrm{pH}=8$ y $\mathrm{pH}=12$ con F.I. entre $0.1 \mathrm{M}-0.5 \mathrm{M}$. Por lo tanto se encontró una remoción del $99.7 \%$ a pH $=12$ y F.I. $=0.1 \mathrm{M}$.

Recientemente Vijayaraghavan y Shanthakumar (2016) removieron Rcongo con un extracto algal proveniente de Sargassum sp. que se asemeja al ALG. Sin embargo, al comparar los espectros FTIR del ALG y el extracto algal (Fan et al. 2005, Caykara y Demirci 2006, Lawrie et al. 2007), se puede concluir que son productos distintos con similares eficiencias de remoción. Además, estos investigadores utilizaron al material como agente coagulante, y en nuestro caso, el ALG adsorbe y atrapa moléculas de colorante cuando forma el ALG (Ca) (Lozano-Álvarez et al. 2015). Esto demuestra el potencial de las algas pardas para aportar materiales que remueven colorantes.

El comportamiento del Rcongo fue similar al anterior colorante, a pesar de una alta repulsión entre el ALG y el colorante por el $\mathrm{pH}$ básico utilizado. No obstante, la formación de agregados por el Rcongo (cuya geometría es plana), los puentes de 
(a)

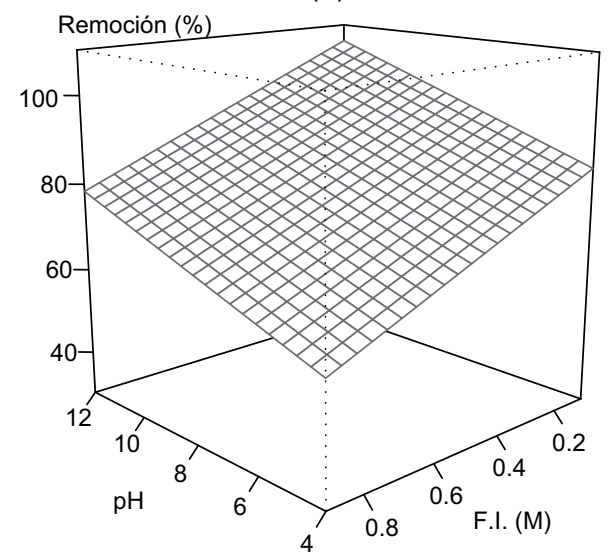

(b)

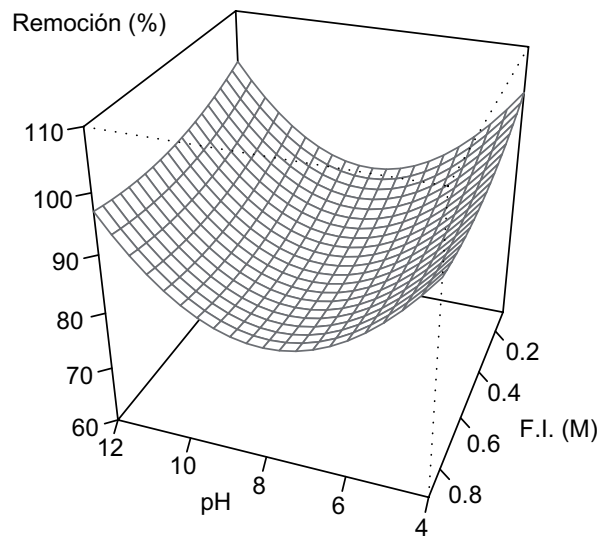

(c)

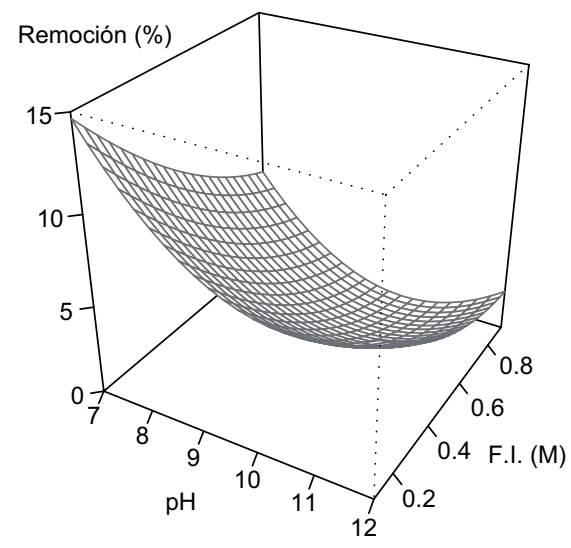

Fig. 3. Eficiencia de remoción de colorantes con ALG en función del pH y la fuerza iónica en un ambiente acuoso: a) DR80, b) Rcongo y c) Rmetilo

hidrógeno entre sus grupos funcionales (azo, amino y sulfonato) y los grupos hidroxilo y carboxilo del ALG además de las interacciones hidrofóbicas entre ambos compuestos, favorecieron una alta remoción del Rcongo. Aunque las moléculas de este colorante son más pequeñas que las del RD80, se agregan de manera muy eficiente (Skowronek 1998), resultando un porcentaje de remoción de $99.7 \%$.

El efecto del pH y la F.I. en la eficiencia de remoción del Rmetilo es representado en la figura 3c. Se observa que la eficiencia de remoción disminuye al incrementarse el $\mathrm{pH}$, consecuencia de un incremento en la repulsión entre el ALG y el Rmetilo por la desprotonación de estos dos compuestos. Por otra parte, al aumentar la F.I. se observa una disminución en la remoción del Rmetilo, debido a una disminución de la interacción entre las moléculas de colorante y el ALG por una gran cantidad de iones $\mathrm{Na}^{+} \mathrm{y} \mathrm{Cl}^{-}$. Además, la figura 3c sugiere que los puentes de hidrógeno entre los grupos funcionales del Rmetilo y el ALG son menos eficientes que los formados por el RD80 y el Rcongo con este biopolímero. Freeman y Mock (2012) reportaron que los grupos sulfonato de diferentes colorantes interaccionan más eficientemente con los grupos hidroxilo del algodón y la celulosa que el grupo carboxilo. Aunque el ALG posee grupos carboxilo, su estructura es abundante en grupos hidroxilo, y se asemeja a estos polisacáridos. Esto explicaría la baja afinidad del Rmetilo por el ALG. Además, el Rmetilo es más pequeño que los anteriores colorantes y tiene menos tendencia a agregarse por la disminución en su planaridad molecular a $\mathrm{pH}$ $=7$ por la predominancia de la especie aniónica (Park et al. 2004). Lo anterior resulta en un eficiencia de remoción del $14.9 \%$ a $\mathrm{pH}=7$ y F.I. $=0.1 \mathrm{M}$.

\section{Obtención de las isotermas de remoción}

Los modelos de Langmuir (1916) y Freundlich (1906) han sido utilizados para describir la remoción de colorantes con distintos materiales (Kyzas y 
Kostoglou 2014). Recientemente Lozano-Álvarez et al. (2009) aplicaron estos modelos y encontraron que dichos modelos no describieron las isotermas experimentales. Por esta razón no se abordó su aplicación a los sistemas estudiados en este trabajo. Zimm y Bragg (1959) propusieron su modelo para describir los cambios conformacionales en proteínas. Recientemente se ha aplicado este modelo para describir la unión de colorantes a ALG y xantana. Dicho modelo considera que las moléculas de colorante se unen a la superficie polimérica y favorecen la unión de moléculas presentes en disolución (fenómeno descrito por $K_{\mathrm{u}}$ o constante de nucleación). Del mismo modo, las moléculas de colorante una vez unidas al polímero tienden a formar agregados (situación descrita por $U$ o constante de cooperatividad). Ambos $K_{\mathrm{u}}$ y $U$ se obtienen por optimización (Lozano-Álvarez et al. 2009, Jáuregui-Rincón et al. 2011). Estos parámetros determinan el valor de $\beta$, que es definida como el número de sitios del polímero ocupados por moléculas de colorante, expresada como una fracción y que está dado por la ecuación:

$$
\beta=\frac{1}{2}\left\{1+\frac{s-1}{\left[(1-s)^{2}+\left(\frac{4 s}{u}\right)\right]^{\frac{1}{2}}}\right\}
$$

donde $\mathrm{s}=K_{\mathrm{u}}$ Ce y Ce representa la concentración de colorante en el equilibrio ( $\mathrm{mol} / \mathrm{L})$.

Conociendo los valores de $U$ y $K_{\mathrm{u}}$, se obtiene la isoterma teórica y se compara con la obtenida experimentalmente. El cuadro III muestra los valores de $K_{\mathrm{u}}$ y $U$ para los diferentes sistemas ALG-colorante y la figura 4 representa las isotermas experimentales y las curvas teóricas respectivas.

Se observa que los sistemas ALG-RD80 (Ca) y ALG-Rcongo (Ca) tienen valores de $K_{\mathrm{u}}$ y $U$ dentro

CUADRO III. CONSTANTES DEL MODELO DE ZIMMBRAGG PARA LOS DIFERENTES SISTEMAS ALGINATO-COLORANTE ESTUDIADOS

\begin{tabular}{lccc}
\hline Sistema & $\begin{array}{c}\text { ALG-DR80 } \\
(\mathrm{Ca})\end{array}$ & $\begin{array}{c}\text { ALG-Rcongo } \\
(\mathrm{Ca})\end{array}$ & $\begin{array}{c}\text { ALG-Rmetilo } \\
(\mathrm{Ca})\end{array}$ \\
\hline$K_{\mathrm{u}}(\mathrm{Kg} / \mathrm{mol})$ & 275463.13 & 324019.41 & 449.00 \\
$U$ & 12.73 & 10.39 & 7.290 \\
$\mathrm{R}^{2}$ & 0.9571 & 0.8700 & 0.6961 \\
$\mathrm{ERP}$ & 21.59 & 21.33 & 39.11 \\
\hline
\end{tabular}

$\mathrm{ERP}=$ error relativo promedio (en porcentaje)

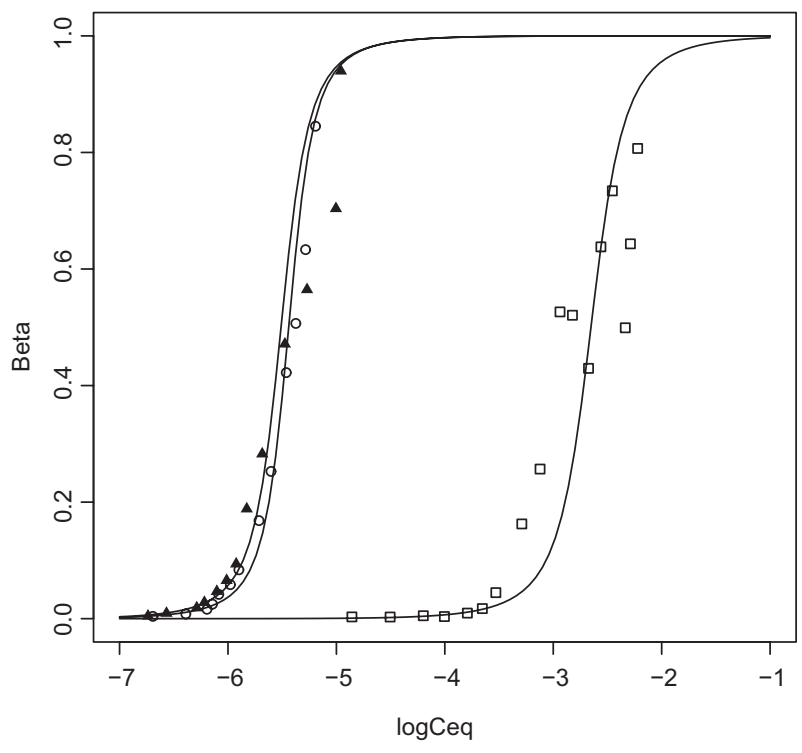

Fig. 4. Datos experimentales e isotermas obtenidas utilizando el modelo de Zimm-Bragg de los sistemas: ALG-DR80 (Ca) (o), ALG-Rcongo (Ca) ( $\mathbf{\Delta}$ ) y ALG-Rmetilo (Ca) (口)

del mismo orden de magnitud y eso se refleja en sus eficiencias de remoción superiores al $99 \%$. En contraparte el ALG-Rmetilo (Ca) muestra los valores más pequeños de dichas constantes, observándose que la isoterma experimental y la teórica se encuentran en el intervalo de Ce entre $10^{-2}$ y $10^{-4} \mathrm{M}$, indicando que en este sistema el ALG remueve menos colorante. Es importante mencionar que el grado de dispersión obtenido en los datos experimentales en todos los sistemas es causado por la desviación de la radiación por el ALG (puesto que es un hidrocoloide) y particularmente en el sistema ALG-Rmetilo (Ca), por la alta sensibilidad del Rmetilo a ligeros cambios de $\mathrm{pH}$.

Considerando a otros colorantes, como el negro directo 22 (DB22), azul directo 1 (DB1), rojo directo 81 (DR81) y naranja de metilo (Nmetilo) (LozanoÁlvarez et al. 2015), se obtuvo el cuadro IV. El RD80 es el colorante de peso molecular mayor, que contiene el mayor número de grupos fenilo, además de poseer dos grupos naftilo, obteniéndose una capacidad de agregación considerable y en un porcentaje de remoción del $99.9 \%$. Nótese que a pesar de la carga negativa del $\mathrm{RD} 80$ a $\mathrm{pH}=12$, sus grupos funcionales (azo, sulfonato, hidroxilo y amido) forman puentes de hidrógeno con el ALG. El segundo colorante de mayor peso molecular (PM) es el DB22, cuya remoción fue del $99.7 \%$, debido a que la atracción electrostática fue la principal fuerza 
CUADRO IV. EFICIENCIAS DE REMOCIÓN DE DIFERENTES COLORANTES AZO Y ALGUNAS DE SUS PROPIEDADES FÍSICAS Y QUÍMICAS

\begin{tabular}{|c|c|c|c|c|c|}
\hline Colorante & $\begin{array}{l}\text { Peso molecular } \\
\qquad \mathrm{g} / \mathrm{mol})\end{array}$ & $\begin{array}{c}\% \\
\text { Remoción }\end{array}$ & $\begin{array}{l}\text { No. de anillos fenilo } \\
\text { (F) y naftilo (N) }\end{array}$ & $\begin{array}{l}\text { Grupos funcionales } \\
\text { presentes }\end{array}$ & Carga \\
\hline DR80 & 1373.07 & 99.9 & $4 \mathrm{~F}$ y $2 \mathrm{~N}$ & Azo, $-\mathrm{SO}_{3},-\mathrm{OH}$, amido & $-8(\mathrm{pH}=12,0.1 \mathrm{M})$ \\
\hline DB22* & 1083.97 & 99.7 & $2 \mathrm{~F}$ y $3 \mathrm{~N}$ & $\mathrm{Azo},-\mathrm{SO}_{3},-\mathrm{OH}$, amido & $+2(\mathrm{pH}=4,0.5-0.9 \mathrm{M})$ \\
\hline Rcongo & 696.66 & 99.7 & $2 \mathrm{~F}$ y $2 \mathrm{~N}$ & Azo, $-\mathrm{NH}_{2}$ y $-\mathrm{SO}_{3}$ & $-2(\mathrm{pH}=12,0.1 \mathrm{M})$ \\
\hline $\mathrm{DB} 1 *$ & 992.80 & 64.0 & $2 \mathrm{~F}$ y $2 \mathrm{~N}$ & Azo $,-\mathrm{NH}_{2},-\mathrm{SO}_{3}$ y $\mathrm{C}=\mathrm{O}$ & $-6(\mathrm{pH}=12,0.9 \mathrm{M})$ \\
\hline DR81* & 675.61 & 41.0 & $2 \mathrm{~F}$ y $1 \mathrm{~N}$ & Azo, $-\mathrm{SO}_{3},-\mathrm{OH}$, amido & $-1(\mathrm{pH}=4,0.1 \mathrm{M})$ \\
\hline Nmetilo & 327.33 & 48.1 & $2 \mathrm{~F}$ & Azo, $-\mathrm{N}\left(\mathrm{CH}_{3}\right)_{2},-\mathrm{SO}_{3}$ & $-1(\mathrm{pH}=12,0.1 \mathrm{M})$ \\
\hline RMetilo & 291.28 & 14.9 & $2 \mathrm{~F}$ & Azo, $-\mathrm{COOH}$ y $-\mathrm{N}\left(\mathrm{CH}_{3}\right)_{2}$ & Aprox. neutra $(\mathrm{pH}=7,0.1 \mathrm{M})$ \\
\hline
\end{tabular}

*Datos tomados de Lozano-Álvarez et al. (2015)

de unión en el ALG-DB22 (Ca) y a que contiene dos grupos fenilo y tres grupos naftilo.

Los datos del cuadro IV indican de manera general que al ir disminuyendo el PM de los colorantes y su capacidad de agregación (dada por la extensión de los anillos aromáticos), su eficiencia de remoción se ve reducida. A excepción del Rcongo que contiene una menor carga, pero una alta planaridad en su geometría molecular (Han et al. 2008) y puede formar estructuras supramoleculares más eficientemente que el DB1 (Roterman et al. 1998). La reducción en el número de anillos aromáticos (Nmetilo y Rmetilo) disminuyó su capacidad de agregación y en consecuencia sus eficiencias de remoción. Más aún, la presencia de un grupo sulfonato en posición para, con respecto al grupo azo en el Nmetilo le permite unirse eficientemente a polisacáridos (Clark 2011). Contrariamente, el Rmetilo posee un grupo carboxilo en posición orto, que lo hace menos afín al ALG, obteniéndose una eficiencia de remoción (14.9\%) menor a la del Nmetilo (48.1\%).

Lo anterior sugiere que la eficiencia de remoción se incrementa a medida que el PM y el número de anillos aromáticos aumentan (dado que la planaridad molecular se favorecerá y se promoverá una alta agregación). Esto se ilustra claramente, si se compara al Rmetilo $(\mathrm{PM}=291.28 \mathrm{~g} / \mathrm{mol} \mathrm{y}$ dos grupos naftilo) con el RD80 (PM $=1373.07 \mathrm{~g} / \mathrm{mol}$ y 4 grupos fenilo y 2 naftilo), cuyas eficiencias de remoción son del $14.9 \%$ y $99.9 \%$ respectivamente. Además una mayor carga positiva promoverá una fuerte atracción hacia el ALG, contribuyendo a una mayor eficiencia de remoción. Esta información permitirá predecir cualitativamente a partir de las estructuras de los colorantes azo, cuál de ellos será removido más eficientemente con el ALG en un ambiente acuoso.

\section{CONCLUSIONES}

Existe una relación cualitativa entre las características estructurales de los colorantes azo y su eficiencia de remoción del medio acuoso por el ALG cuando se consideran moléculas con PM que van desde $291.28 \mathrm{~g} / \mathrm{mol}$ (Rmetilo) hasta PM de 1373.07 $\mathrm{g} / \mathrm{mol}$ (RD80). Las moléculas con mayor PM, una mayor carga positiva y una eficiente capacidad de agregación, producen valores más altos en su eficiencia de remoción. De acuerdo con esto se puede predecir cualitativamente, a partir de la estructura de los colorantes azo, si un colorante se removerá más eficientemente que otro al utilizar al ALG.

Es necesario seguir generando más información para soportar esta relación de manera más sólida, más aún, la extensión de esta investigación a otros tipos de colorantes como los reactivos, básicos, ácidos, dispersos, etc., ayudará a un entendimiento mayor de las interacciones entre este polisacárido y las moléculas de colorante. Lo anterior permitirá desarrollar la tecnología adecuada para la remoción de colorantes de diferentes efluentes acuosos y disminuirá la contaminación de cuerpos de agua mediante el uso de polímeros biodegradables como el alginato de sodio.

\section{REFERENCIAS}

Abbott L.C., Batchelor S.N., Jansen L., Oakes J., Lindsay Smith J.R. y Moore J.N. (2011). Picosecond deactivation of azo dye excited states in solution and in cellulose. J. Photochem. Photobiol. A Chem. 218 (1), 11-16. DOI: $10.1016 /$ j.jphotochem.2010.11.017

Anjaneyulu Y., Chary N.S. y Suman-Raj D.S. (2005) Decolourization of industrial effluents - available methods 
and emerging technologies - a review. Rev. Environ. Sci. Biotechnol. 14 (4), 245-273.

DOI: $10.1007 / \mathrm{s} 11157-005-1246-\mathrm{z}$

Armstrong D.R., Clarkson J. y Smith W.E. (1995). Vibrational analysis of trans-azobenzene. J. Phys. Chem. 99 (51), 17825-17831. DOI: 10.1021/j100051a005

Barkauskas J., Stankeviciene I., Daksevic J. y Padarauskas A. (2011). Interaction between graphite oxide and congo red in aqueous media. Carbon. 49 (15), 53735381. DOI: 10.1016/j.carbon.2011.08.004

Bhatnagar A. y Sillanpää M. (2009). Applications of chitinand chitosan-derivatives for the detoxification of water and wastewater -A short review. Adv. Colloid Interface Sci. 152 (1-2), 26-38. DOI: 10.1016/j.cis.2009.09.003

Biswas N. y Umapathy S. (2000). Structures, vibrational frequencies, and normal modes of substituted azo dyes: infrared, Raman, and density functional calculations.

J. Phys. Chem. A. 104 (12), 2734-2745.

DOI: $10.1021 /$ jp9929263

Blackburn R.S. (2004). Natural polysaccharides and their interactions with dyes molecules: applications in effluent treatment. Environ. Sci. Technol. 38 (18), 4905-4909.

DOI: $10.1021 / \mathrm{es} 049972 \mathrm{n}$

Bonancea C.E., do Nascimento G.M., de Souza M.L., Temperini M.L.A. y Corio P. (2006). Substrate development for surface-enhanced Raman study of photocatalytic degradation processes: congo red over silver modified titanium dioxide films. Appl. Catal. B. 69 (1-2), 34-42. DOI: 10.1016/j.apcatb.2006.05.016

Caykara T.C. y Demirci S. (2006). Preparation and characterization of blend films of poly (vinyl alcohol) and sodium alginate. J. Macromol. Sci. A. 43 (7), 11131121. DOI: 10.1080/10601320600740389

Clark M. (2011). Handbook of textile and industrial dyeing, Volume 1: Principles, processes and types of dyes. Woodhead Publishing, EUA, 652 pp.

Core R. T. R. (2013). A language and environment for statistical computing [en línea]. http://www.R-project. org 06/02/2017

Draget K.I. (2009). Alginates. En: Handbook of hydrocolloids. (G.O. Phillips y P.A. Williams, Eds.). CRC Press, Boca Raton, EUA, pp. 807-828.

DOI: $10.1533 / 9781845695873.807$

Elhaddaoui A., Merlin J.C., Delacourte A. y Turrell S. (1992). Spectroscopic studies of the effect of $\mathrm{pH}$ on the interaction between congo red and amyloid-type proteins J. Mol. Struct. 267, 113-116. DOI: 10.1016/0022-2860(92)87018-Q

Fan L., Du Y., Zhang B., Yang J., Cai J., Zhang L. y Zhou J. (2005). Preparation and properties of alginate/watersoluble chitin blend fibers. J. Macromol. Sci. A. 42 (6), 723-732. DOI: 10.1081/MA-200058635
Freeman H.S. y Mock G.N. (2012). Dye application, manufacture of dye intermediates and dyes. En: Handbook of industrial chemistry and biotechnology. (J.A. Kent, Ed.), Springer, Boston, EUA, pp. 475-548.

DOI: $10.1007 / 978-1-4614-4259-2 \_13$

Freundlich H.M.F. (1906). Uber die adsorption in Losungen Z. Phys. Chem. 57, 385-471.

DOI: $10.1515 /$ zpch-1907-5723

García-Heras M., Kromka K., Faber J., Karaszkiewics P. y Villegas M.A. (2005). Evaluation of air acidity through optical sensors. Environ. Sci. Technol. 39 (10), 37433747. DOI: 10.1021/es049558n

Ghorai S., Sarkar A., Raoufi M., Panda A. B., Schönherr H. y Pal S. (2014). Enhanced removal of methylene blue and methyl violet dyes from aqueous solution using a nanocomposite of hydrolyzed polyacrylamide grafted xanthan gum and incorporated nanosilica. ACS Appl. Mater. Interfaces. 6 (7), 4766-4777.

DOI: $10.1021 / \mathrm{am} 4055657$

Gupta V.K. y Suhas. (2009). Application of low-cost adsorbents for dye removal-A review. J. Environ. Manage. 90 (8), 2313-2342. DOI: 10.1016/j.jenvman.2008.11.017

Haldorai Y. y Shim J.J. (2014). An efficient removal of methyl orange dye from aqueous solution by adsorption onto chitosan/ $\mathrm{MgO}$ composite: A novel reusable adsorbent. Appl. Surf. Sci. 292, 447-453.

DOI: 10.1016/j.apsusc.2013.11.158

Han R., Ding D., Xu Y., Zou W.,Wang Y., Li Y. y Zou L. (2008). Use of rice husk for the adsorption of congo red from aqueous solution in column mode. Biores. Technol. 99 (8), 2938-2946.

DOI: 10.1016/j.biortech.2007.06.027

Ho Y.S. y McKay G. (1999). Pseudosecond order model for sorption processes. Process Biochem. 34 (5), 451465. DOI: 10.1016/S0032-9592(98)00112-5

Jáuregui-Rincón J., Lozano-Álvarez J. y Medina-Ramírez I. (2011). Zimm-Bragg model applied to sorption of dyes by biopolymers: alginic acid and xanthan. En: Biotechnology of biopolymers. (M. Elnashar, Ed.). Intech, Croacia, pp. 165-190. DOI: 10.5772/17074

Karadag E., Ödemis H., Kundakci S. y Üzum O.B. (2016). Swelling characterization of acrylamide/zinc acrylate/ xanthan gum/sepiolite hybrid hydrogels and Its application in sorption of janus green B from aqueous solutions. Adv. Polym. Technol. 35 (3), 248-259.

DOI: $10.1002 / \mathrm{adv} .21547$

Karukstis K.K., Perelman L.A. y Wong W.K. (2002). Spectroscopic characterization of azo dyes aggregation on dendrimer surfaces. Langmuir 18 (26), 10363-10371. DOI: $10.1021 / 1 \mathrm{a} 020558 \mathrm{f}$

Kyzas G.Z. y Kostoglou M. (2014). Green adsorbents for wastewaters: A critical review. Materials 7 (1), 333364. DOI: $10.3390 / \mathrm{ma} 7010333$ 
Lawrie G., Keen I., Drew B., Chandler-Temple A., Rintoul L., Fredericks P. y Grøndahl L. (2007). Interactions between alginate and chitosan biopolymers characterized using FTIR and XPS. Biomacromolecules 8 (8), 2533-2541. DOI: 10.1021/bm070014y

Langmuir I. (1916). The Constitution and fundamental properties of solids and liquids J. Am. Chem. Soc. 38, 2221-2295.DOI: 10.1021/ja02268a002

Lozano-Álvarez J.A., Jáuregui-Rincón J., Mendoza-Díaz G., Rodríguez-Vázquez R. y Frausto-Reyes C. (2009). Study of sorption equilibrium of biopolymers alginic acid and xanthan with C.I. disperse yellow 54. J. Mex. Chem Soc. 53 (2), 59-70.

Lozano-Álvarez J.A., Marañón-Ruíz V.F., JáureguiRincón J., Medina-Ramírez I., Frausto-Reyes C. y Salinas-Gutiérrez R. (2015). Removal of direct dyes with alginic acid. J. Mex. Chem. Soc. 59 (3), 215-227.

Mittal H., Parashar V., Mishra S.B. y Mishra A.K. (2014). $\mathrm{Fe}_{3} \mathrm{O}_{4} \mathrm{MNPs}$ and gum xanthan based hydrogels nanocomposites for the efficient capture of malachite green from aqueous solution. Chem. Eng. J. 255, 471-482. DOI: 10.1016/j.cej.2014.04.098

Movahedi M., Mahjoub A.R. y Janitabar-Darzi S. (2009). Photodegradation of congo red in aqueous solution on $\mathrm{ZnO}$ as an alternative catalyst to $\mathrm{TiO}_{2}$. J. Iran. Chem. Soc. 6 (3), 570-577. DOI: 10.1007/BF03246536

Oakes J. y Dixon S. (2004). Physical interactions of dyes in solution-influence of dye structure on aggregation and binding to surfactants/polymers. Rev. Prog. Color. 34 (1), 110-128.

DOI: 10.1111/j.1478-4408.2004.tb00156.x

Oakes J. y Gratton P. (1998). Kinetic investigations of azo dye oxidation in aqueous media J. Chem. Soc., Perkin Trans. 2 (9), 1857-1864. DOI: 10.1039/A803892I

Oller I., Malato S. y Sánchez-Pérez J.A. (2011). Combination of advanced oxidation processes and biological treatments for wastewater decontamination - A review. Sci. Total Environ. 409 (20), 4141-4166.

DOI: 10.1016/j.scitotenv.2010.08.061

Ortiz-Monsalve S., Dornellesa J., Poll E., RamírezCastrillón M., Valentec P. y Gutierres M. (2017). Biodecolourisation and biodegradation of leather dyes by a native isolate of Trametes villosa. Process Saf. Environ. 109, 437-451.

DOI: $10.1016 /$ j.psep.2017.04.028

Ozen A. S., Doruker P. y Aviyente V. (2007). Effect of cooperative hydrogen bonding in azo-hydrazone tautomerism of azo dyes J. Phys. Chem. A. 111 (51), 13506-13514. DOI: 10.1021/jp0755645

Pal M. K y Mandal N. (1990). Binding of cationic dyes to potassium alginate: A spectrophotometric and dichroic probe. Biopolymers 29 (12-13), 1541-1548.

DOI: 10.1002/bip.360291205
Park S.K., Lee C., Min K.C. y Lee N.S. (2004). Fourier transform Raman studies of methyl red adsorbed on $\gamma$-alumina and silica-alumina Bull. Korean Chem. Soc. 25 (12), 1817-1821.

DOI: $10.5012 /$ bkcs.2004.25.12.1817

Robinson T., McMullan G., Marchant R. y Nigam P. (2001). Remediation of dyes in textile effluent: a critical review on current treatment technologies with a proposed alternative. Bioresour. Technol. 77 (3), 247255. DOI: $10.1016 / \mathrm{S} 0960-8524(00) 00080-8$

Roterman I., Rybarska J., Konieczny L., Skowronek M., Stopa B., Piekarska B. y Bakalarski G. (1998). Congo red bound to $\alpha-1$-proteinase inhibitor as a model of supramolecular ligand and protein complex. Computers Chem. 22 (1), 61-70.

DOI: $10.1016 / \mathrm{S} 0097-8485(97) 00014-4$

Sabnis R.W. (2007). Handbook of acid-base indicators, CRC Press, Boca Raton, EUA 398 pp.

Sadeghi-Kiakhani M., Arami M. y Gharanjig, K. (2013). Preparation of chitosan-ethyl acrylate as a biopolymer adsorbent for basic dyes removal from colored solutions. J Environ. Chem. Eng. 1 (3), 406-415.

DOI: $10.1016 /$ j.jece.2013.06.001

Saratale R.G., Saratale G.D., Chang J.S. y Govindwar S.P. (2011). Bacterial decolorization and degradation of azo dyes: A review. J. Taiwan Inst. Chem. E. 42 (1), 138-157. DOI: $10.1016 /$ j.jtice.2010.06.006

Schrader B. (1995). Vibrational spectroscopy of different classes and states of compounds. En: Infrared and Raman spectroscopy, methods and applications, (B. Schrader, Ed.). VCH Verlagsgesellschaft $\mathrm{mbH}$, Germany, 189-222.

DOI: $10.1002 / 9783527615438$

Silverstein R.M., Webster F.X., Kiemle D.J. y Bryce D.L. (2015). Spectrometric identification of organic compounds. 8a. ed. John Wiley and Sons, EUA, 453 pp.

Skowronek M., Stopa B., Konieczny L., Rybarska J., Piekarska B., Szneler E. Bakalarski G. I. y Roterman I. (1998). Self-assembly of congo red-a theoretical and experimental approach to identify its supramolecular organization in water and salt solutions. Biopolymers 46 (5), 267-281. DOI: 10.1002/(SICI)10970282(19981015)46:5<267::AID-BIP1>3.0.CO;2-N

Solís M., Solís A., Pérez H.I., Manjarrez N. y Flores M. (2012). Microbial decolouration of azo dyes: a review. Process Biochem. 47 (12), 1723-1748.

DOI: $10.1016 /$ j.procbio.2012.08.014

Song J., Ma K., Jiao T., Xing R., Zhang L., Zhou J. y Peng Q. (2017). Preparation and self-assembly of graphene oxide-dye composite Langmuir films: nanostructures and aggregations. Colloids Surf. A Physicochem. Eng. Asp. 529, 793-800.

DOI: $10.1016 /$ j.colsurfa.2017.06.071 
Srinivasan A. y Viraraghavan T. (2010). Decolorization of dye wastewaters by biosorbents: A review. J. Environ. Manage. 91 (10), 1915-1929.

DOI: $10.1016 /$ j.jenvman.2010.05.003

Vijayaraghavan G. y Shanthakumar S. (2016). Performance study on algal alginate as natural coagulant for the removal of congo red dye. Desalin. Water Treat. 57 (14), 6384-6392. DOI: 10. 1021/ed035p514
Xu Y., Lebrun R.E., Gallo P.J. y Blond P. (1999). Treatment of textile dye plant effluent by nanofiltration membrane. Separ. Sci. Technol. 34 (13), 2501-2519. DOI: $10.1081 / \mathrm{SS}-100100787$

Zimm B.H. y Bragg J.K. (1959). Theory of phase transition between helix and helix coil in polypeptide chains. J. Chem. Phys. 31 (2), 526-535. DOI: 10.1063/1.1730390 